\title{
The Cologne Database for Molecular Spectroscopy, CDMS, in the Virtual Atomic and Molecular Data Centre, VAMDC
}

\author{
Christian P. Endres ${ }^{\mathrm{a}, 1}$, Stephan Schlemmer ${ }^{\mathrm{a}}$, Peter Schilke ${ }^{\mathrm{a}}$, Jürgen Stutzki ${ }^{\mathrm{a}}$, Holger S.P. Müller ${ }^{\mathrm{a}, *}$ \\ ${ }^{a}$ I. Physikalisches Institut, Universität zu Köln, Zülpicher Str. 77, 50937 Köln, Germany
}

\begin{abstract}
The Cologne Database for Molecular Spectroscopy, CDMS, was founded 1998 to provide in its catalog section line lists of mostly molecular species which are or may be observed in various astronomical sources by means of (usually) radio astronomical means. The line lists contain transition frequencies with qualified accuracies, intensities, quantum numbers, as well as further auxilary information. They have been generated from critically evaluated experimental line lists, mostly from laboratory experiments, employing established Hamiltonian models. Seperate entries exist for different isotopic species and usually also for different vibrational states. As of December 2015, the number of entries is 792. They are available online as ascii tables with additional files documenting information on the entries.

The Virtual Atomic and Molecular Data Centre, VAMDC, was founded more than 5 years ago as a common platform for atomic and molecular data. This platform facilitates exchange not only between spectroscopic databases related to astrophysics or astrochemistry, but also with collisional and kinetic databases. A dedicated infrastructure was developed to provide a common data format in the various databases enabling queries to a large variety of databases on atomic and molecular data at once.

For CDMS, the incorporation in VAMDC was combined with several modifications on the generation of CDMS catalog entries. Here we introduce related changes to the data structure and the data content in the CDMS. The new data scheme allows us to incorporate all previous data entries but in addition allows us also to include entries based on new theoretical descriptions. Moreover, the CDMS entries have been transferred into a mySQL database format. These developments within the VAMDC framework have in part been driven by the needs of the astronomical community to be able to deal efficiently with large data sets obtained with the Herschel Space Telescope or, more recently, with the Atacama Large Millimeter Array.
\end{abstract}

Keywords:

database, line identification, rotational spectroscopy, Hamiltonian, radio astronomy, astrochemistry

\section{Introduction}

The unambiguous assignment of a spectral line feature observed in space to a specific molecular or atomic species and its quantitative interpretation, e.g. in terms of column densities or relative abundances, depends decisively on the availability of rest frequencies and intensity information with sufficient accuracy. Such information is usually generated from laboratory data, sometimes supplemented by astronomical observations for rest frequencies and quite often by quantum chemical calculations for intensity information. The reliability of the derived frequencies and intensities depends on the quality of the Hamiltonian model, the accuracies of the rest frequencies and of the intensity information, but also very much on the proper judgement of the line frequency accuracies. We will discuss important aspects on this topic in section 2

\footnotetext{
${ }^{*}$ Corresponding author.

Email address: hspm@ph1.uni-koeln.de (Holger S.P. Müller)

${ }^{1}$ Present address: Max-Planck-Institut für extraterrestrische Physik, Giessenbachstrasse 1, 85748 Garching, Germany
} 
The CDMS2 [1, 2] was founded in the year 1998 to provide such information in its catalog section following the example of and complementing the JPL catalog $3[3]$ because of limited activities in the JPL catalog in recent years. The emphasis in the CDMS catalog is put on molecular species of interest for astrophysics or astrochemistry which have been or may be observed by radio astronomical means. The catalog also contains entries for fine structure transitions of atoms, such as $\mathrm{C},{ }^{13} \mathrm{C}, \mathrm{C}^{+},{ }^{13} \mathrm{C}^{+}, \mathrm{N}^{+}, \mathrm{O}, \mathrm{Al}$ etc. In selected cases, rovibrational transitions are available not only in the submillimeter (sub-mm) or far-infrared (FIR) region, such as for $\mathrm{C}_{3}$ or $\mathrm{C}_{3} \mathrm{O}_{2}$, but sometimes also in the mid-IR, such as for $\mathrm{CH}^{+}, \mathrm{CCH}, \mathrm{HCO}^{+}$, and $\mathrm{N}_{2} \mathrm{H}^{+}$. Some of the species are also important for studies of planetary atmospheres, including that of Earth, for example $\mathrm{HCN}, \mathrm{H}_{2} \mathrm{CO}, \mathrm{PH}_{3}, \mathrm{CH}_{3} \mathrm{CN}$, and $\mathrm{SO}_{2}$. Individual entries are created for different isotopologues as far as they may be relevant for astronomers. In addition, transitions of different vibrational states are usually provided in different entries as well; they may be in one single entry for simple diatomics, such as $\mathrm{NaCl}$ and $\mathrm{KCl}$, or strongly coupled vibrational states. The number of entries approaches 800 at the end of 2015; an up-to-date list is available onlinet. These entries, together with those in the JPL catalog, cover a large fraction of the almost 200 molecules detected in the interstellar medium (ISM) or in circumstellar envelopes (CSEs) 5 of late type stars with minor isotopologues or excited vibrational states. In fact, the CDMS and JPL catalogs were instrumental for the analyses of spectral recordings obtained, e.g., with the Herschel Space Telescope. In addition, data collections, such as the Leiden Atomic and Molecular DAtabase (LAMDA) [4], CASSIS[5], and splatalogue [6, 7], draw heavily on the entries of the CDMS and JPL catalogs. Other databases, such as GEISA [8, 9] and HITRAN [10], use many data from the CDMS and JPL databases, in particular in their pure rotational parts.

The line list entries are generated from spectroscopic parameters, their uncertainties, and their correlation coefficients. These values are obtained from experimental data employing established Hamiltonian models. The experimental data usually stem from laboratory spectroscopic investigations, but data from astronomical observations can also be used as far as appropriate. In most cases, the SPFIT/SPCAT program suite [11] is used for fitting and prediction of molecular spectra. Other programs have been used on occasion, details are provided in section 2.2 .

In the early years of the CDMS, considerable effort had been put on terahertz data of light hydrides, such as entries on $\mathrm{H}_{2} \mathrm{D}^{+}, \mathrm{HD}_{2}^{+}, \mathrm{CH}, \mathrm{CH}^{+}$and its isotopologues, $\mathrm{CH}_{2}, \mathrm{NH}, \mathrm{ND}, \mathrm{NH}_{2},{ }^{15} \mathrm{NH}_{3}, \mathrm{OH}^{+}, \mathrm{H}_{2} \mathrm{O}^{+}, \mathrm{SH}^{+}$, and $\mathrm{H}_{2} \mathrm{~S}, \mathrm{H}_{2} \mathrm{Cl}^{+}$, and $\mathrm{ArH}^{+}$along with their isotopologues. The aim was to support missions such as the Herschel Space Telescope [12], especially with respect to its high resolution Heterodyne Instrument for the Far-Infrared, HIFI, [13] and the Stratospheric Observatory For Infrared Astronomy, and SOFIA, [14] with its German REceiver for Astronomy at Terahertz frequencies, GREAT, [15]. Herschel in particular has expanded our knowledge in the field of terahertz astronomy tremendously [16], in particular with respect to hydrides. One of the main goals were low-energy rotational transitions of $\mathrm{H}_{2} \mathrm{O}$ and its isotopologues [17]. Large scale molecular line surveys were another important goal. They led to the detection of several hydrides in Galactic or extragalactic sources, the latest detection being $\mathrm{ArH}^{+}[18$, 19], a tracer of the almost completely atomic ISM, that was also detected in an extragalactic source recently with ALMA [20]. Important discoveries with SOFIA/GREAT include the detection of SH [21] and of the ground state rotational transition of para $-\mathrm{H}_{2} \mathrm{D}^{+}$; the latter observation benefitted from earlier laboratory work [23], see also section 2.1. Terahertz observation are possible to some extent even from the ground, as demonstrated, e.g., by the early observations of $\mathrm{OH}^{+}$[24] and $\mathrm{SH}^{+}$[25] with the Atacama Pathfinder EXperiment, APEX, [26].

The focus of new or updated entries in the CDMS has shifted somewhat with the advent of the Atacama Large Millimeter Array, ALMA. Due to the very high sensitivity and high spatial resolution, which reduces confusion caused by multiple source components in the telescope beam, interferometers are superior to single dish telescopes in detecting complex organic molecules as exemplified by the detection of iso-propyl cyanide $\left(i-\mathrm{C}_{3} \mathrm{H}_{7} \mathrm{CN}\right)$ as the first branched alkyl compound [27] whose detection was only possible because of fairly recent laboratory work [28]. Transitions belonging to vibrationally excited states are easier to detect, e.g. highly excited HNC toward the carbonrich late-type star CW Leonis [29]; such transitions provide deeper insight into excitation mechanisms in general and insight into the dust formation zone in the particular case of late-type stars. Interferometric observations of late-type stars provide considerably more detail than single dish observations and can lead to the detection of new species, such as the recent detection of $\mathrm{TiO}_{2}$ and the radio astronomical detection of $\mathrm{TiO}$ in the circumstellar envelope of the O-rich

\footnotetext{
${ }^{2}$ http://www.astro.uni-koeln.de/cdms/

${ }^{3} \mathrm{http}: / /$ spec.jpl.nasa.gov/

${ }^{4} \mathrm{http}: / /$ www.astro.uni-koeln.de/cdms/entries

${ }^{5}$ See, e.g., http://www.astro.uni-koeln.de/cdms/molecules
} 
AGB star VY Canis Majoris [30]. $\mathrm{TiO}_{2}$ was also observed with ALMA during science verification observations [31]. Interferometric observations will also be very beneficial for the investigations of extragalactic sources, such as the unnamed foreground galaxy in the direction of the quasar PKS 1830-211 [32], in which also $\mathrm{ArH}^{+}$was detected [20].

Radio astronomical spectra are often analyzed under the assumption of local thermodynamic equilibrium (LTE). This is usually a good assumption in warm and dense parts of molecular clouds, but deviations from LTE may be considerable at lower temperatures, e.g. in dark clouds, or in less dense regions, especially in the diffuse ISM. In these cases it is often necessary to take into account collisional processes with $\mathrm{H}_{2}$ and $\mathrm{He}$ in the denser ISM, and/or with $\mathrm{H}$ and electrons in the more diffuse ISM. A review on collisional processes in the ISM has been published fairly recently [33]. Accurate data are needed for a plethora of molecules to avoid misinterpretations. An interesting and important aspect was the detection of seemingly more $\mathrm{HNC}$ than the more stable $\mathrm{HCN}$. These results were obtained by assuming the somewhat similar molecular structure would lead to similar collisional rates with $\mathrm{He}$ and $\mathrm{H}_{2}$. In the absence of collisional data for $\mathrm{HNC}$, those of $\mathrm{HCN}$ were used instead. Initial calculations of $\mathrm{HNC}$ with $\mathrm{He}$ and later with $\mathrm{H}_{2}$ [34] revealed that the collisional rates are in fact very different and the column densities of $\mathrm{HNC}$ had to be revised to values lower than those of $\mathrm{HCN}$ in all instances.

Collisional rates are available from laboratory experiments for some systems. In many cases, however, such data are not available, and often it may be difficult to obtain such data. Resorting to quantum chemical calculations is a common alternative. But the calculation of collisional processes by quantum chemical means with high accuracy is demanding even nowadays. Therefore, calculations are often restricted to He as the collider and rates of collisions with $\mathrm{H}_{2}$ are often estimated from these. Also, these calculations consider mostly collisons with small molecules, which play a more important role in cold or less dense environments than complex organic molecules. Neverteless, the need for collisional data involving more complex molecules has become inceasingly apparent in recent years. The complex absorption and emission spectrum of methyl formate $\left(\mathrm{CH}_{3} \mathrm{OCHO}\right)$ in the GBT PRIMOS survey of Sagittarius B2(N) between $\sim 0.3$ and $\sim 50 \mathrm{GHz}$ is an interesting case because the observed low energy transitions sample the less dense envelope of this source, and their intensity distribution, including weak maser activity in some lines, could not be explained by an LTE model [35].

The need to access an increasing number of accurate molecular spectroscopy data together with an increasing number of accurate collisional data has become apparent in recent years. An early effort to link molecular spectroscopy data (here from the CDMS and JPL catalogs) with collisional data in a virtual observatory compliant environment was undertaken within the BASECOL collisional data base [36, 37]. Eventually, this effort led to the Virtual Atomic and Molecular Data Centre (VAMDC) [38], an infrastructure which links further collisional databases, molecular spectroscopy databases in other frequency regions (e.g. databases such as HITRAN [10] or ExoMol [39]), atomic spectroscopy databases, and also kinetic databases such as KIDA [40] and UMIST [41]. The CDMS has participated in the VAMDC from the beginning, and one main part of this article, section 3 , describes changes in the content of the CDMS catalog that were required for reliable data exchange between the various VAMDC partners, but also changes that have been made to serve the users of the CDMS catalog.

As a consequence of supporting VAMDC's infrastructure, the CDMS has undergone a technical transformation process. The data are stored in a relational database (mysql) and standards, such as query languages to access the database, and output formats, which are developed within VAMDC will be supported. This process comes along with an improvement of the provided data in terms of their physical meaning and description. Besides simplifying the use and access of the data, one of the goals is also to make the data origin more transparent and enable others to reproduce the data. This aspect is described in section 4

\section{Considerations for creating CDMS catalog entries}

The generation of CDMS catalog entries requires several steps. The first one is the decision that the spectrum of a (mostly) molecular species is or may be of interest for the community that uses the CDMS catalog or the decision that an existing entry requires update because of the need and availability of additional, usually new, data of sufficient impact. Collecting available experimental line lists may be anything from very straightforward to timeconsuming, as can be the case for the subsequent steps. These are the generation of Hamiltonian models, evaluation

${ }^{6}$ http://www.vamdc.eu/; http://www.vamdc.org/ 
of intensity-related aspects, such as dipole moment components and partition functions, and finally the generation of a documentation which provides background information on the entry.

\subsection{Aspects related to experimental line lists}

Line lists of rotational data may consist of just a single line, but may also be extensive, especially in cases of molecules with a complex spectrum, for example because of fine or hyperfine structure splitting, such as the $\mathrm{NH}_{2}$ radical. A large data set from diverse sources was assembled in Ref. [42]. In cases like this one, it is particularly important to evaluate the correctness of reported line frequency uncertainties and estimate values in cases in which no uncertainties have been reported. Since this process is not independent of the choice of Hamiltonian parameters, an iterative process may be required. In the end, it is not only desirable that the entire experimental line list has been reproduced within ascribed uncertainties on average, but this should also be tested for reasonably well defined subsets. Details are again available for $\mathrm{NH}_{2}$ [42]. Another example of a CDMS related publication is the fit of radio-frequency to terahertz data of several NO isotopologues plus heterodyne IR data for the main species [43].

The inclusion of rovibrational or rovibronic data in a fit is particularly important if the data known with microwave accuracy are very limited. Only the $J^{\prime \prime}=0$ (i.e., $J=1-0$ ) rotational transition frequencies of $\mathrm{CH}^{+},{ }^{13} \mathrm{CH}^{+}$, and $\mathrm{CD}^{+}$have been published thus far [44]. These data were combined subsequently with extensive rovibronic data of the $A-X$ electronic transitions of four isotopologues to evaluate rotational and rovibrational transition frequencies [45]. The prediction of rotational transitions of the main isotopologue were accurate enough for analysis of emission spectra taken with the moderate resolution PACS instrument on board the Herschel satellite toward a disk around Herbig Be star HD 100546 which extended to $J^{\prime \prime}=5$ [46]. Another example is argonium. Extensive rotational and rovibrational data are available for ${ }^{40} \mathrm{ArH}^{+}$and ${ }^{40} \mathrm{ArD}^{+}$because ${ }^{40} \mathrm{Ar}$ (from the radioactive decay of ${ }^{40} \mathrm{~K}$ ) is by far the most abundant argon isotope on Earth. In the Solar atmosphere and in the interstellar medium, ${ }^{36} \mathrm{Ar}$ is most abundant, with ${ }^{38} \mathrm{Ar}$ lower by a factor of around five, and ${ }^{40} \mathrm{Ar}$ being largely negligible. The only published data with microwave accuracy involving isotopologues containing ${ }^{36} \mathrm{Ar}$ or ${ }^{38} \mathrm{Ar}$ are the $J^{\prime \prime}=0$ transition frequencies of ${ }^{36} \mathrm{ArD}^{+}$and ${ }^{38} \mathrm{ArD}^{+}$ [47]. Combining these data with the extensive rotational and rovibrational data for ${ }^{40} \mathrm{ArH}^{+}$and ${ }^{40} \mathrm{ArD}^{+}$yielded CDMS entries that facilitated the identification of the $J^{\prime \prime}=0$ and 1 transitions of ${ }^{36} \mathrm{ArH}^{+}$in emission in the Crab Nebula [19], the $J^{\prime \prime}=0$ transition of ${ }^{36} \mathrm{ArH}^{+}$in absorption in the diffuse medium toward several continuum sources, of the same transition of ${ }^{38} \mathrm{ArH}^{+}$toward Sagittarius B2(M) [18], and of the $J=1-0$ transitions of ${ }^{36} \mathrm{ArH}^{+}$and ${ }^{38} \mathrm{ArH}^{+}$in a $z=0.89$ foreground galaxy in absorption toward the quasar PKS 1830-211 [20]. Several cationic molecules, such as $\mathrm{CH}^{+}$or argonium, occur mostly or exclusively in the diffuse interstellar medium because in the dense medium they are readily destroyed by reaction with $\mathrm{H}_{2}$. Excitation of such molecular cations by collision with $\mathrm{H}_{2}$ can not be invoked to explain observation of some of the cations in emission under specific circumstances; instead, excitation through collisions with electrons, as evaluated, e.g., in Ref. [48], or with $\mathrm{H}$ atoms can explain such observations. In the case of sparse rotational data, accurate ground-state combination differences from IR spectra can be very useful also, such as recently determined for isotopic species of $\mathrm{H}_{3}^{+}$[49].

Experimental lines from astronomical observations can also be very useful, in particular for molecules that are difficult to synthesize in the laboratory. In some cases, initial assignments were based on astronomical data only, such as in the cases of $\mathrm{C}_{5} \mathrm{~N}^{-}[50]$ and $\mathrm{C}_{3} \mathrm{H}^{+}$[51]. Laboratory rotational data have been obtained for the latter cation in the meantime [52, 53], in great part because of the dispute concerning the carrier of the lines. Data from astronomical observations can even be important if they only supplement laboratory data and if they have lower accuracies, such as in the case of extensive Herschel-HIFI data on the cyclic $\mathrm{SiC}_{2}$ molecule [54].

The correctness, i.e., the accuracy and precision, of a reported transition frequency is an important aspect for molecules with small line list. In the case of rotational ground state transitions of $\mathrm{H}_{2} \mathrm{D}^{+}\left[23\right.$ ] or $\mathrm{CH}^{+}$[44] errors of $\sim 60 \mathrm{MHz}$ were revealed with respect to earlier reports. Frequency errors may also be found in the course of astronomical observation, such as the case of the lowest fine structure component lines of $\mathrm{SH}^{+}$near $346 \mathrm{GHz}$ [55]. Subsequent laboratory measurements provided improved accuracies for these lines as well as remeasurements of the fine structure component lines near $526 \mathrm{GHz}$ along with two of the four hyperfine lines of the highest fine structure component of the $N=1-0$ transition [56]. Such errors are easier to identify in larger data sets, where, in addition, the omission of a single line has frequently a small effect.

The uncertainties of experimental lines in a fit are of major concern. Earlier publications frequently refrained from quoting any specific value, but this has changed considerably in recent years. Nevertheless, a recent paper quotes uncertainties of better than $50 \mathrm{kHz}$ up to $500 \mathrm{kHz}$ for a very large data set. This information, however, is not particularly 
useful if no uncertainties appear in the line list. Sometimes uncertainties of previously reported data get modified without justification and without apparent reason. We have found quite frequently that the reported transition frequency uncertainties were judged too conservatively or too optimistically. The uncertainties of the resulting spectroscopic parameters may be somewhat reasonable if so-called standard errors are reported. However, the uncertainties of the lines are still incorrect, and the lines may carry a too large or too small weight in more extensive line lists. It is always much better to try to evaluate appropriate uncertainties; this is often not too difficult if the line list is sufficiently large and the Hamiltonian model is complete.

\subsection{Hamiltonian models}

CDMS catalog entries are mostly generated employing Pickett's SPFIT/SPCAT program suite [11], a versatile program developed to fit asymmetric rotors with multiple vibrational states or nuclear or electronic spins. The program evolved with time [57, 58]. Extended treatment of symmetry and spin-statistics [59] and the introduction of Euler functions for fitting spectra of moderately rigid molecules, such as $\mathrm{H}_{2} \mathrm{O}$ [60] were major extensions of the program. Special considerations exist for linear molecules and symmetric tops, especially for $l$-doubled states. Quantum numbers as well as the number of vibrational states can exceed 100 by far.

The choice of spectroscopic parameters to be used in a fit is often far from unique. This is particularly the case if the primary objective to include a reasonable parameter in a fit is its determination with significance. Such approach leads too easily to too large parameter sets in cases of complex spectra such as the rotation-tunneling spectrum of the lowest energy conformer of ethanediol [61] or the Coriolis interaction between $v_{4}=1$ and $v_{6}=1$ of $\mathrm{ClClO}_{2}$ [62]. Searching at all stages of the fit among the reasonable spectroscopic parameters for the one that reduces the root mean squares (rms) error of the fit most appears to be a suitable strategy to keep the number of spectroscopic parameters in the fit small. The importance of the choice of reductions and representations have been discussed often, Ref. [63] is an example in which other aspects (e.g. condition numbers) have also been discussed.

In multi-state fits, there are different ways to reduce the number of spectroscopic parameters similar to the familiar Dunham expansion for diatomic molecules. Such approaches can be applied to fitting of rotation-tunneling spectra, as in the cases of ethanediol [61], $\mathrm{H}_{2} \mathrm{DO}^{+}[64]$, and ethanethiol [65]. In the case of gauche $-\mathrm{C}_{2} \mathrm{H}_{5} \mathrm{SH}$, the number of spectroscopic parameters was reduced to 34 from 41 in a traditional two-state fit [66].

The combination of diverse data pertaining to one molecular species can improve the spectroscopic parameters considerably. This is often not only beneficial from the molecular physics point of view, but also for the predictions to be used for radio astronomical observations; some examples are $\mathrm{CH}^{+}$[45], $\mathrm{H}_{3} \mathrm{O}^{+}$[67], $\mathrm{H}_{2} \mathrm{DO}^{+}[64]$, and $\mathrm{PH}_{3}$ [68].

A careful choice of spectroscopic parameters has led in some cases to predictions that turned out in later investigations to be considerably better than those based on original publications, as in the cases of oxirane [69] and $\mathrm{CHD}_{2} \mathrm{CN}$ [70]. Technical aspects of a fitting program may also lead to incorrect Hamiltonian parameters, as has happened for $\mathrm{NaCN}$ [71].

We point out that there are entries in the CDMS catalog which have not been generated with SPFIT/SPCAT. This applies, in particular, to molecules which display splitting caused by large amplitude motions such as the internal rotation of one or more $\mathrm{CH}_{3}$ groups. Several programs are available, as described in a fairly recent review [72]. Existing entries in the CDMS catalog include $\mathrm{CH}_{3} \mathrm{OH}$ and ${ }^{13} \mathrm{CH}_{3} \mathrm{OH}$ [73], dimethyl ether [74], $\mathrm{CH}_{3} \mathrm{SH}_{\text {[75] }}, \mathrm{CH}_{3}^{18} \mathrm{OH}$ [76], and $\mathrm{CH}_{3} \mathrm{O}^{13} \mathrm{CHO}[77]$.

\subsection{Dipole moments and intensities}

Dipole moment components of a given molecule are important pieces of information to calculate the intensity of a rotational transition at a certain temperature, its line strength $S \mu^{2}$, where $S$ is the intrinsic line strength, and $\mu$ is the effective dipole moment component, or its Einstein $A$ value 7 (e.g. [3]). They can be determined from Stark effect measurements which have been quite common in times of Stark-modulation rotational spectroscopy, but are less common nowadays. Nevertheless, new measurements include $\mathrm{TiO}_{2}$ [78], 1,2-propanediol [79], and 1,3-propanediol [80]. Occasionally, dipole moment components get reinvestigated, as in the cases of the lowest energy conformer of n-propanol [81], iso-propyl cyanide [28], and ethyl and vinyl cyanide [82]. Such measurements usually improve older

\footnotetext{
${ }^{7}$ http://www.astro.uni-koeln.de/cdms/catalog\#equations
} 
measurements considerable, but in some cases, one or more dipole moment components get revised considerably such as in the cases of $i s o$-propyl cyanide [28] and vinyl cyanide [82].

Stark effect measurements seek in most cases to resolve the individual Stark components as well as possible. An opposite approach does work, however. In the weak field limit, one seeks to keep all Stark components within a single and symmetric line and measures only a small Stark shift of some tens of kilohertz; this can lead to dipole moment components accurate at the percent level [83].

Quantum chemical calculations of dipole moment components are an important alternative for experimental determinations. In some cases, even selections of several molecules are studied to support the astronomical community [84, 85]. Such calculations [86] are even more important for transition dipole moments of not so stable molecules because reliable intensity measurements may be difficult or even impossible.

Rotational, also know as centrifugal distortion, and other corrections may have to be taken into account in particular for rotational spectra of light hydrides or for molecules with large amplitude motions. They may also matter for rigid molecules in highly rotationally excited transitions. In cases of mixing of states mediated by internal rotation, tunneling, or vibration-rotation interaction, sign choices of spectroscopic parameters, which describe the mixing, relative to those of the dipole moment components may have significant impact on the intensities of some transitions. Examples have been given for the lowest energy conformer of ethanediol [61] or $\mathrm{H}_{2} \mathrm{DO}^{+}$[64]. Recently, intensity problems in the rotation-tunneling spectrum of ethanol in the $3 \mathrm{~mm}$ wavelength region became apparent by astronomical observations with ALMA and were, at least largely, resolved by a change of the sign of one dipole moment component [65].

\subsection{Partition functions}

The partition function $Q$ is very important to evaluate intensities of molecular or atomic lines assuming thermal population or to derive column densities. In the usual case, in which entries have been generated with the SPCAT progam [11], partition function values at selected temperatures are calculated directly by summation over the energy levels. These values, sometime supplemented by additional values at other, in particular lower or higher temperatures, are provided in the documentation file, see section 2.5 , a separate page ${ }^{8}$ lists the decadic logarithm of these values. The partition function can be separated to first order into a rotational part $\left(Q_{\text {rot }}\right)$ and a vibrational part $\left(Q_{\text {vib }}\right)$. Traditionally, the CDMS as well as the JPL catalog were usually restricted to $\left(Q_{\text {rot }}\right)$ only, i.e., they have provided partition function values for the ground vibrational state only. More complete partition function values have become available in more recent entries.

The Boltzmann constant used for evaluation of the partition function is about $2 \times 10^{-6}$ larger than the 2010 CODATA value [87]; this has a negligible effect on the calculated intensities. In addition, uncertainties in the partition function because of limitations in the accuracy of spectroscopic parameters, in particular the vibrational energies, see below, are usually much larger also, except for some diatomics.

If the ground state partition function values are determined by numerical summation over the energies of a particular species, it is important that the maximum $J$ (or $F$ ) and $K$ values have been chosen high enough to ensure convergence of the partition function values at $300 \mathrm{~K}$. The SPCAT program calculates these values down to units of $10^{-4}$. The CDMS entries have usually been generated applying a partition function value that is converged to that level or at least nearly so. In the case of the light $\mathrm{CH}$ radical, this is achieved at $F=15$. In case of the still light HCN molecule, $J=49$ is required. $\mathrm{HC}_{3} \mathrm{~N}$ and $\mathrm{HC}_{5} \mathrm{~N}$ require $J=152$ and 298 , respectively. However, this criterion is rather strict because the CDMS catalog provides the decadic logarithm of the intensity at $300 \mathrm{~K}$ given to four digits after the decimal point, thus a $Q(300)$ value too low by a factor of 1.000115 affects the decadic logarithm of the intensity at best in the last digit given. In the case of $\mathrm{HC}_{5} \mathrm{~N}$, this means that $J=206$ is already sufficient for proper calculation of the decadic logarithm of the intensity to all quoted digits in most cases. Easing the accuracy requirements to $1 \%$ and $10 \%$ reduces the maximum $J$ value to be considered to 147 and 103 , respectively.

Calculating the partition function values up to $300 \mathrm{~K}$ or even higher by summation over the energies may not be always feasible, in particular for very large molecules or for molecules with a very complex Hamiltonian. Treating the molecule as a rigid rotor is a very efficient alternative; a better compromise could be to calculate the partition function by summation over the energies up to a certain temperature and correct the rigid rotor approximation for higher

\footnotetext{
${ }^{8}$ http://www.astro.uni-koeln.de/site/vorhersagen/catalog/partition_function.html
} 
temperatures by extrapolation from lower temperature deviations. These approaches are good or at least reasonable as long as the molecule is not too floppy.

Care is advised if the partition function is calculated by summation over the energies if there are relatively large distortion parameters in the Hamiltonian. For example, if $D_{K}$ or $L_{K}$ is the highest order purely $K$-dependent distortion term in an asymmetric rotor close to the prolate limit, higher $K$ levels will be lower in energy than lower ones eventually, and the energies may eventually even turn negative. This is one important reason why maximum $J$ and $K$ quantum numbers should not be chosen much higher than needed.

Assuming thermal population, excited vibrational states may even be populated at temperatures just barely larger than those of dark clouds. At $300 \mathrm{~K}$, a vibration with vibrational energy of $480 \mathrm{~cm}^{-1}$ or $691 \mathrm{~K}$ is down in population by a factor of 0.1 with respect to the ground state. At twice the energy or half the temperature, the population is down by a factor of 0.01. Larger organic molecules may have low-lying vibrations (torsional modes or bending modes) which may well be below $100 \mathrm{~cm}^{-1}$ or $144 \mathrm{~K}$. In addition, they may also have more than one low-lying conformation. In the case of, e.g., ethanol and $n$-propanol, the combined vibrational and conformation factors at $150 \mathrm{~K}$ were evaluated to be 2.96 and $\sim 5.2$ if the respective data set is restricted to the ground vibrational state of the lowest energy conformer only [65]. Vibrational corrections to the partition function, and hence to the column density, are difficult to evaluate because in particular low-lying vibrational states (in the FIR region) are often not known or only known with limited accuracy because the transitions are weak, only rather low-resolution measurements were made, or the measurements were not made in the gas phase. Additional complications may arise from incorrectly reported experimental data, such as in the case of vinyl cyanide, in which the energy of $v_{10}$ was revised from 788 to $561 \mathrm{~cm}^{-1}$ [90]. Quantumchemical calculations on vibrational properties at diverse levels of theory are quite common nowadays. High-level calculations are often very demanding on larger molecular systems, and the resulting accuracy may still be limited. A combination of experimental and quantum-chemical data may be a good compromise in many cases. Experimentally, the anharmonic fundamental vibrations are determined to a varying extent. Quantum-chemical calculations provide in most instances the harmonic vibrations. Detailed information on the anharmonicity is rarely available. Nevertheless, estimating contributions from overtone and combination states via the harmonic oscillator approximation provide better estimates of the vibrational contributions than the restriction to known vibrational levels. Various hybrid ways of evaluating vibrational contributions can be made. In Ref. [88], the rotational part of the partition function was converged up to room temperature. Vibrational contributions up to $v_{8}=3$ at almost $1100 \mathrm{~cm}^{-1}$ were taken into account directly; the truncation error was estimated to be $1.408 \%$ at $296 \mathrm{~K}$ via the harmonic oscillator approximation. A different type of mixed approach was taken for three isotopologues of methyl formate [89]. Torsional contributions up to $v_{\mathrm{t}}=6$ were evaluated from data up to $v_{\mathrm{t}}=2$, and contributions from the other vibrations were estimated by the harmonic oscillator approximation. For larger molecules, a fully classical calculation of the partition function may be the most reasonable way. This was done, e.g., for the acetone entry in the JPL catalog, a fairly heavy molecule with two very low lying torsional modes and a rather low CCC bending mode.

For the calculation of astrophysical line intensities, one should keep in mind that a significant uncertainty arises from the fact that in real molecular clouds high vibrational states are not necessarily in LTE with the gas temperature, and that, therefore, the partition function calculated under this assumption will be erroneous. In many cases, vibrational states are excited by absorption of IR radiation, and their excitation temperature is therefore determined by the effective radiation temperature of the IR, which is not necessarily equal to the kinetic temperature which will determine the LTE of the vibrational ground state through collisions. The details will depend on the exact source structure, and would need to be determined by exact radiative transfer calculations.

\subsection{Documentation files}

For each catalog entry, there is a documentation which provides basic information on the content of the entry. These are the chemical formula, the name (possibly alternative names), the symmetry of the (usually ground) electronic state, and possibly vibrational information, the tag to identfy the species, the number of lines (i.e. transition frequencies), the highest quantum number, and the highest frequency. The references, from which the experimental transition frequencies were taken, are listed usually completely, though some early entries may list references only in part. Information on the treatment of the data may also be given, such as omission of data, judgment of uncertainties, or information on the Hamiltonian model. Other information includes estimates on the reliability of the entry with respect to the needs of astronomers, partition function values, often also information on limitations of the partition function (e.g., the usual limitation to the ground vibrational state, or the consideration of vibrational states), rotational 
constants, dipole moment components, among other information. Links to special entries, for example with or without HFS, special spin states, etc., may also be given. The consideration of non-trivial spin-statistics is generally mentioned as well.

\section{Content of the VAMDC-CDMS}

The main focus of the CDMS database is providing accurate transition frequencies for astronomical use. This biases the selection of molecular and atomic species and the selection of vibrational states. Usually, experimental data reported in the literature have been fit to an effective Hamiltonian. Subsequently, the derived parameters are used to calculate transition frequencies, intensities as well as state energies.

The data are organized in entries which are identified by a unique, sequential identifier which replaces the formerly used species tag. Different atomic and molecular species are assigned to different entries, but one entry comprises at most data stemming from the same compilation. This means the data from one entry have been fit together and assures thereby that the data in themselves are consistent. Data from the same compilation have been distributed into two or more entries in several cases. This may be the case if more than one vibrational state have been involved.

Each entry contains information on radiative transitions, state energies, partition functions, origin of the data and a documentation. Data are only provided if transition frequencies are known accurately enough to be useful for astronomical observations; this means if the rest frequencies are known better than one $\mathrm{MHz}$ and on average better than $100 \mathrm{kHz}$. Older versions of entries have been archived in either CDMS version since the start of the VAMDC project. This allows one to review data retrieved earlier and assures backward traceability.

\subsection{Documentation of entries}

Documentation information continues to be available for each entry. The main difference with respect to the single documentation page in the old CDMS implementation is that certain pieces of information are available in separate navigation items. These include a brief description of the data used in the fit and estimates on the reliability of the provided data sets. Basic molecular constants, such as dipole moments and rotational constants, as well as some parameters which have been used for the compilation of the data are given. The files which have been used to prepare the entry are usually provided in order to allow traceability and thereby to improve the quality assurance. Links to cited resources are provided as well as links to other data repositories, such as ChemSpider 19 , NIST Chemistry WebBook 10 , and databases connected to the VAMDC network.

\subsection{Radiative Transitions}

The listings of radiative transitions represents the central part of the database. Rest frequencies, uncertainties, intensities at $300 \mathrm{~K}$, Einstein $A$ values and quantum numbers are given for each listed transition. Upper and lower states are linked in order to provide information on the energy $\left(\mathrm{in}_{\mathrm{cm}}^{-1}\right.$ ) as well as on the degeneracy of the states involved in the transition. Quantum numbers are now reported in physical meaningful and commonly used quantities by applying the standard introduced by VAMDC (see section below). This improves not only the readablility of the quantum numbers, but is also of importance to match state information among different databases. The old ascii format, which is based on the output of the Pickett's SPCAT program, will still be available and stored internally in order to simplify quality assurence and bug tracking, as well as to support third party programs which may rely on the traditional format.

\subsection{State energy listing}

The databases now provide rovibrational state energy listings for each entry. In most cases, the band origin is not as accurately known as the transition frequencies. Absolute values are accurate only if rovibrational transitions have been included in the fit. Usually, the energy of the band center is taken from the literature and absolute uncertainties of one or several wavenumbers are common. The relative values within a vibrational level are, in contrast, as accurate

\footnotetext{
${ }^{9}$ http://www.chemspider.com

${ }^{10} \mathrm{http}: / /$ webbook.nist.gov
} 
as specified, because their values are obtained from the fitted experimental transition frequencies and are thus the basis on which transition frequencies are calculated. Although their absolute values may be known only to about a wavenumber, the state information might still be a reliable basis for collisional data. The state energy listing also constitutes the basis on which the partition functions are calculated.

\subsection{Present considerations of evaluation of partition functions}

Partition functions are provided for 110 temperatures between $1 \mathrm{~K}$ and $1000 \mathrm{~K}$. They are calculated as a sum over all states of a specific species listed in the database. If the data of one species are distributed over more than one entry, which is regularly the case if data for many vibrational states are reported, all states of that specie are linked to each of these entries in order to provide the most complete partition function possible based on the available data (covering all reported states).

$$
Z(T)=\sum_{v} \sum_{\text {rot-states }} g_{I} e^{-E / k_{B} T} \quad, v \text { in CDMS }
$$

with $g_{I}$ the spin-statistical weight, and $E$ the energy of the respective rovibrational level.

Since the state information reported in the database may not be sufficiently comprehensive to allow the calculation of reliable partition functions even at low temperatures for a number of molecules, in particular if low-lying torsional states are present, a routine to calculate the partition function based on the harmonic approximation has been introduced in the database tools and is used to provide more accurate values in such cases if the result of the summation is known to be insufficient. At present, only the harmonic oscillator approximation for asymmetric top molecules is included:

$$
\begin{aligned}
Z_{r o t}(T) & =g_{t o t} T^{1.5} / \sigma \sqrt{\left(\pi(k / h)^{3} /(A B C)\right.} \\
Z_{v i b}(T) & =\prod_{v}\left(1-e^{-E(v) / k T}\right)^{-1} \\
Z(T) & =Z_{r o t}(T) Z_{v i b}(T)
\end{aligned}
$$

where $A, B$, and $C$ are the rotational constants, $\sigma$ is the symmetry number, and $g_{t o t}$ is the total spin statistical weight.

\subsection{References}

Large efforts have been made to improve citations to original data used in the database and to supplement the documentation. VAMDC rules prescribe that references have to be given to all provided values. Thus, each experimental frequency reported in the database includes the reference pointing to the original publication. Nevertheless, some entries remain for which explicit references to experimental frequencies are still missing and are only included in the documentation.

\section{Infrastructure of the VAMDC-CDMS}

A new infrastructure for atomic and molecular data has been developed within the VAMDC framework. CDMS has been migrated over the last couple years to VAMDC and is now available. Standard web-techniques have been applied allowing easy integration of database services into any computer program or webservice. Data access and retrieval is based on the http protocol. Queries to the databases can be formulated already in any web-browser within an URL-string and a large variety of methods is available for almost any computer language to implement this protocol and thus gain access to VAMDC databases. VAMDCs standard data output and data exchange format is VAMDCXSAMS, a modified version of the International Atomic Energy Agency's XML Schema for Atoms, Molecules and Solids (XSAMS) [38]. 


\subsection{Access Protocols and Query/Retrieval Language}

VAMDC uses the web-service protocol VAMDC-TAP for data access services. That means, queries use GET and POST requests to HTTP/HTTPS URLs and the syntax of the URL is defined by the protokol. VAMDC-TAP is a variant of the Table Access Protocol (TAP) defined by IVOA (International Virtual Observatory Alliance) [38]. Each VAMDC-TAP service (database) can return results of queries in VAMDC-XSAMS, supports synchronous data-queries and provides a description of its availability and its service capabilities as specified in TAP by reference to the Virtual Observatory Support Interfaces (VOSI) standard. The query language, which is supported by all VAMDC databases, is VAMDC SQL sub-set \#2 (VSS2). It defines how queries requesting specific data from VAMDC databases have to be formulated. It is similar to the well-known database language SQL. An example VAMDC-TAP request to CDMS querying all data for the $\mathrm{CO}$ molecule could be carried out the following way:

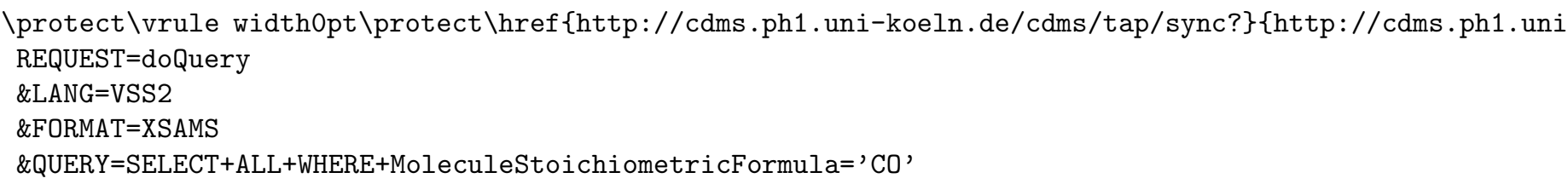

The same request can be sent to any other VAMDC database, just by exchanging the base URL appropriately.

\subsection{Data Models and XML Schema}

The standard model VAMDC-XSAMS is structured in sections specifying species, physical processes, sources, methods, functions, and environments. References to data sources and information on methods used to generate the data is linked to provided data values and are strict requirements to allow traceablility. Physical states of atoms, molecules, ions are described as unambigously and meaningful as possible. Information, such as statistical weights and a reference to the state specifying the energy origin can be attached, as in the following example, which specifies the $J=0$ ground state of the $\mathrm{CO}$ molecule:

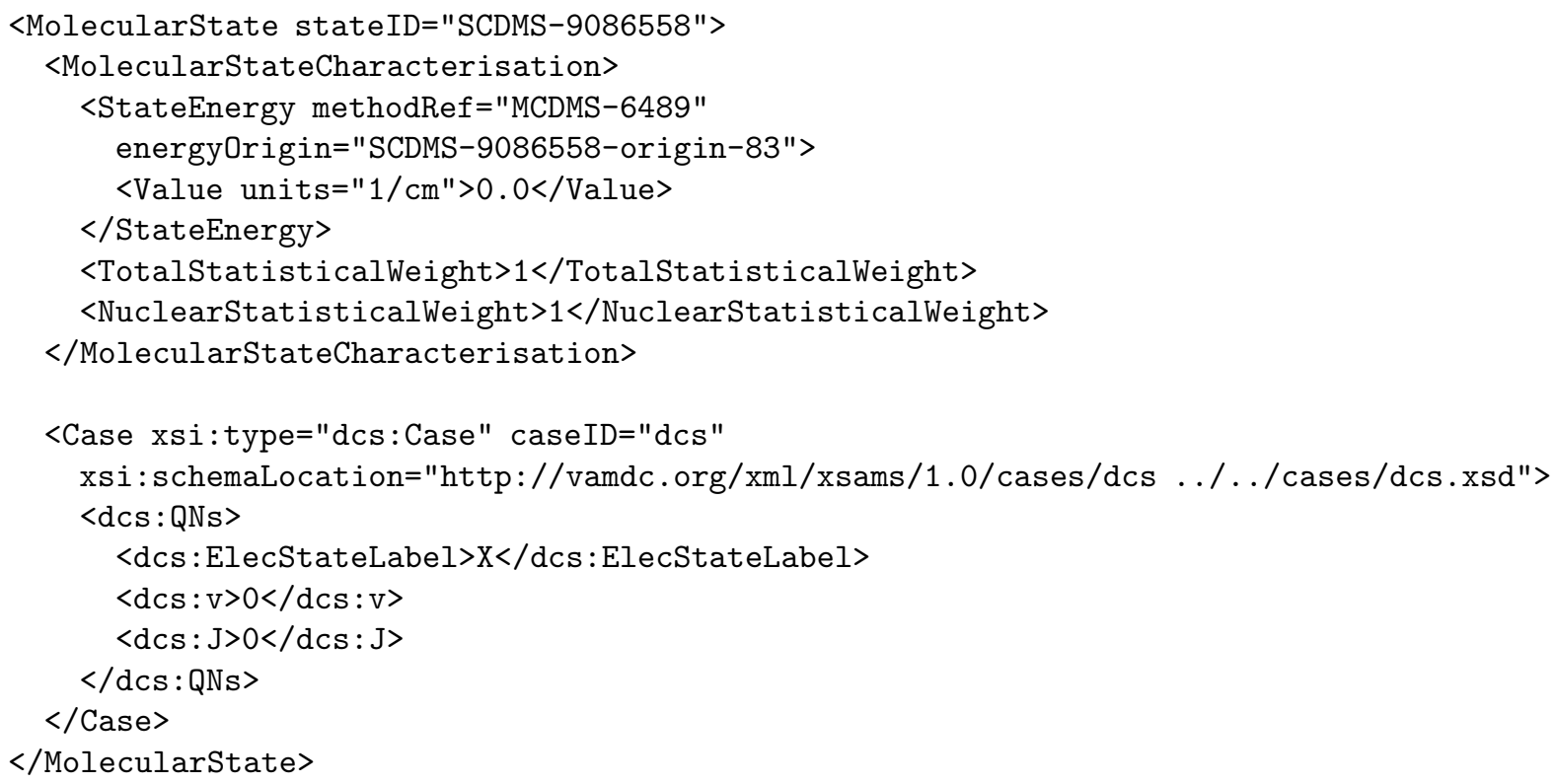

Physical processes are defined in terms of states using a reference to initial and final state as shown in the following example, which describes the rotational transition $J=1-0$ of $\mathrm{CO}$ in the vibrational ground state:

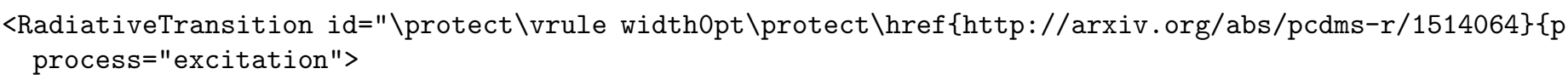




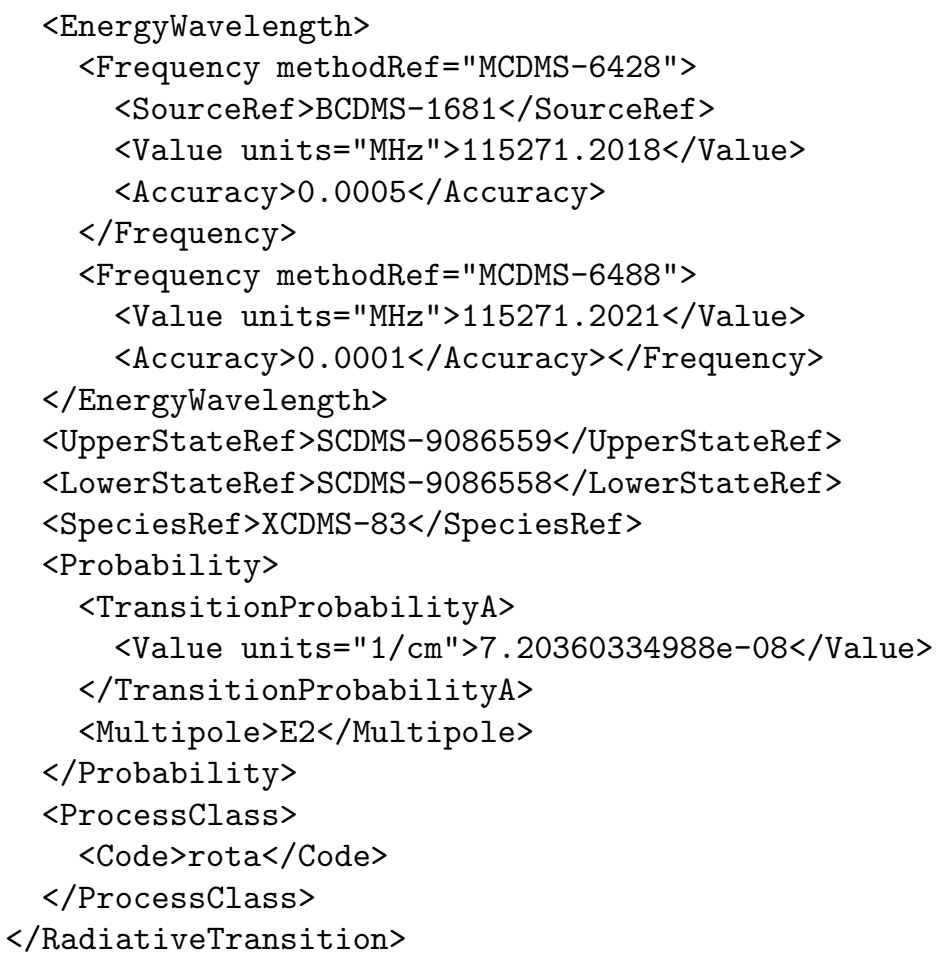

As VAMDC-XSAMS is an XML schema, transformation into any other data format and extraction of data of a user's interest is easy and will also be supported by VAMDC libraries. An example is shown in Fig. 1 The result of a query requesting transitions of the CO molecule via VAMDC-TAP obtained in VAMDC-XSAMS is transformed by the CDMS portal into HTML code.

\subsection{Definition of quantum numbers}

An important aspect of the data exchange format VAMDC-XSAMS is the definition of quantum numbers. A simple, but precise definition of quantum numbers allowing unambigous description of states is key to allow automated cross-correlation of data between various databases. Therefore, different sets of quantum numbers have been defined reflecting the commonly used labeling schemes for all different groups of molecules, such as closed shell diatomic molecules (dcs), open shell asymmetric top molecules (asymos), open shell linear molecules (lpos), for example. Each set contains a sequence of quantum numbers following closely the commonly used notations. Vibrational quantum numbers are accompagnied by the an identifyer of the normal mode associated with it, various parity and symmetry labels are distinguished, and total (or intermediate) angular momentum quantum numbers include an identifier of the nuclear spin associated with it (e.g. $F$, nuclearSpinRef $=$ "N"). The following example gives the definition for the $J_{K_{a}, K_{c}}=1_{1,0}, F=0, s$ inversion state of $\mathrm{NH}_{2} \mathrm{D}$ :

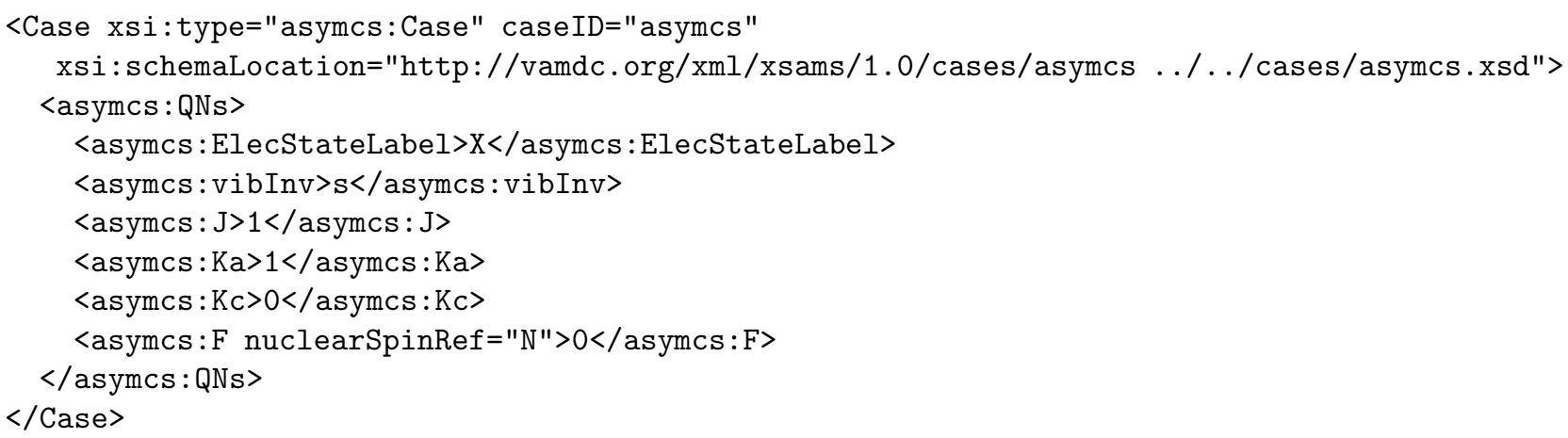




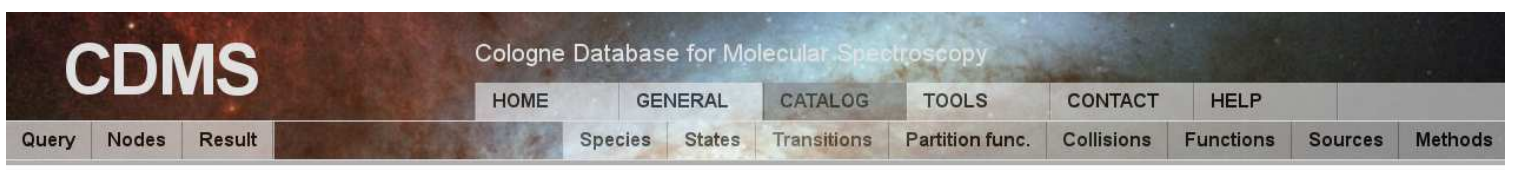

\begin{tabular}{|c|c|c|c|c|c|c|}
\hline 115271.2018 & 0.0005 & 0.00000007 & IS: & 00010.0 & v:0; J:O, & XCDMS-83: CO \\
\hline 115271.2021 & 0.0001 & & F.S: & 00033.845033 & v.0; J:1; & \\
\hline 230538.0000 & 0.0005 & 0.00000069 & IS: & 00033.845033 & v.0; J:1; & XCDMS-83: CO \\
\hline 230538.0000 & 0.0001 & & FS: & 000511.534953 & v.o; J:2, & \\
\hline 345795.9899 & 0.0005 & 0.00000250 & IS: & 000511.534953 & $\mathrm{~V}: 0 ; \mathrm{J}: 2$ & XCDMS- $83: C 0$ \\
\hline 345795.9899 & 0.0002 & & F.S: & 000723.069466 & v.0; J.3 & \\
\hline 461040.7682 & 0.0005 & 0.00000613 & IS: & 000723.069466 & v.0; J.3, & XCDMS-83: CO \\
\hline 461040.7681 & 0.0002 & & F.S: & 000938.448131 & v. $0 ;$ J:4, & \\
\hline 576267.9305 & 0.0005 & 0.00001221 & I.S: & 000938.448131 & v.0; J:4, & XCDMS-83: CO \\
\hline 576267.9310 & 0.0003 & & F.S: & 001157.67036 & v. $0 ; \mathrm{J}: 5$ & \\
\hline 691473.0763 & 0.0005 & 0.00002137 & IS: & 001157.67036 & v. $0 ; \mathrm{J}: 5$ & XCDMS-83: CC \\
\hline 691473.0760 & 0.0003 & & F.S: & 001380.735419 & v. $0 ; \mathrm{J}: 6$ & \\
\hline 806651.8060 & 0.0050 & 0.00003422 & I.S: & 001380.735419 & v. $0 ; \mathrm{J}: 6$ & XCDMS-83: CO \\
\hline 806651.8008 & 0.0004 & & FS: & 0015107.642427 & v. $0 ; \mathrm{J}: 7$ & \\
\hline 921799.7000 & 0.0050 & 0.00005134 & IS: & 0015107.642427 & v. $0 ; \mathrm{J}: 7 ;$ & XCDMS-83: CO \\
\hline 921799.7039 & 0.0005 & & F.S: & 0017138.390355 & v. $0 ; \mathrm{J}: 8$ & \\
\hline 1036912.3930 & 0.0050 & 0.00007330 & IS: & 0017138.390355 & v. $0 ; \mathrm{J}: 8$ & XCDMS-83: CO \\
\hline 1036912.3846 & 0,0007 & & F.S: & 0019172.978029 & ง.0; J:9, & \\
\hline 1151985.4520 & 0.0110 & 0.00010064 & IS: & 0019172.978029 & v.0; J:9, & XCDMS-83: CO \\
\hline 1151985.4434 & 0.0009 & & F.S: & 0021211.404127 & v.0, J:10; & \\
\hline 1267014.4860 & 0.0050 & 0.00013390 & IS: & 0021211.404127 & v. $0 ;$ J:10; & XCDMS-83: CO \\
\hline 1267014.4817 & 0.0012 & & FS: & 0023253.667181 & v.0; J:11; & \\
\hline 1381995.1050 & 0.0130 & 0.00017353 & I.S: & 0023253.667181 & v. $0 ; \mathrm{J}: 11$ & XCDMS-83: CO \\
\hline 1381995.1022 & 0.0015 & & FS: & 0025299.765576 & v. $0 ; \mathrm{J}: 12$ & \\
\hline 1496922.9090 & 0.0120 & 0.00022003 & IS: & 0025299.765576 & v.0; J:12; & XCDMS-83: CC \\
\hline 1496922.9091 & 0,0018 & & F.S: & 0027349.69755 & v. $0 ;$ J:13; & \\
\hline 1611793.5180 & 0.0110 & 0.00027390 & I.S: & 0027349.69755 & v.0; J:13; & XCDMS-83: CO \\
\hline 1611793.5079 & 0.0021 & & F.S: & 0029403.461194 & v.0; J:14; & \\
\hline \multirow[t]{2}{*}{1726602.5057} & 0.0024 & 0.00033535 & IS: & 0029403.461194 & v.0; J:14; & XCDMS-83:CO \\
\hline & & & F.S: & 0031461.054454 & v.0. J:15: & \\
\hline
\end{tabular}

Figure 1: CDMS-Portal - Query page. The page displays information on transitions of the ${ }^{12} \mathrm{C}^{16} \mathrm{O}$ molecule as obtained from CDMS. The data itself is obtained as VAMDC-XSAMS file and transformed by the portal into the form shown above. Frequencies [in MHz], uncertainties [in $\mathrm{MHz}$ ], Einstein $A$ values [in s${ }^{-1}$ ] are provided in the first three columns. Experimental and calculated values are shown in separate rows. The recommended values are shown in bold. References will be shown if the mouse cursor is moved over the displayed value. The next columns contain state information (statistical weight, energy $\left[\right.$ in $\left.\mathrm{cm}^{-1}\right]$ and quantum numbers). Lower state information is displayed in the upper row, whereas upper state information is provided in the lower row. 


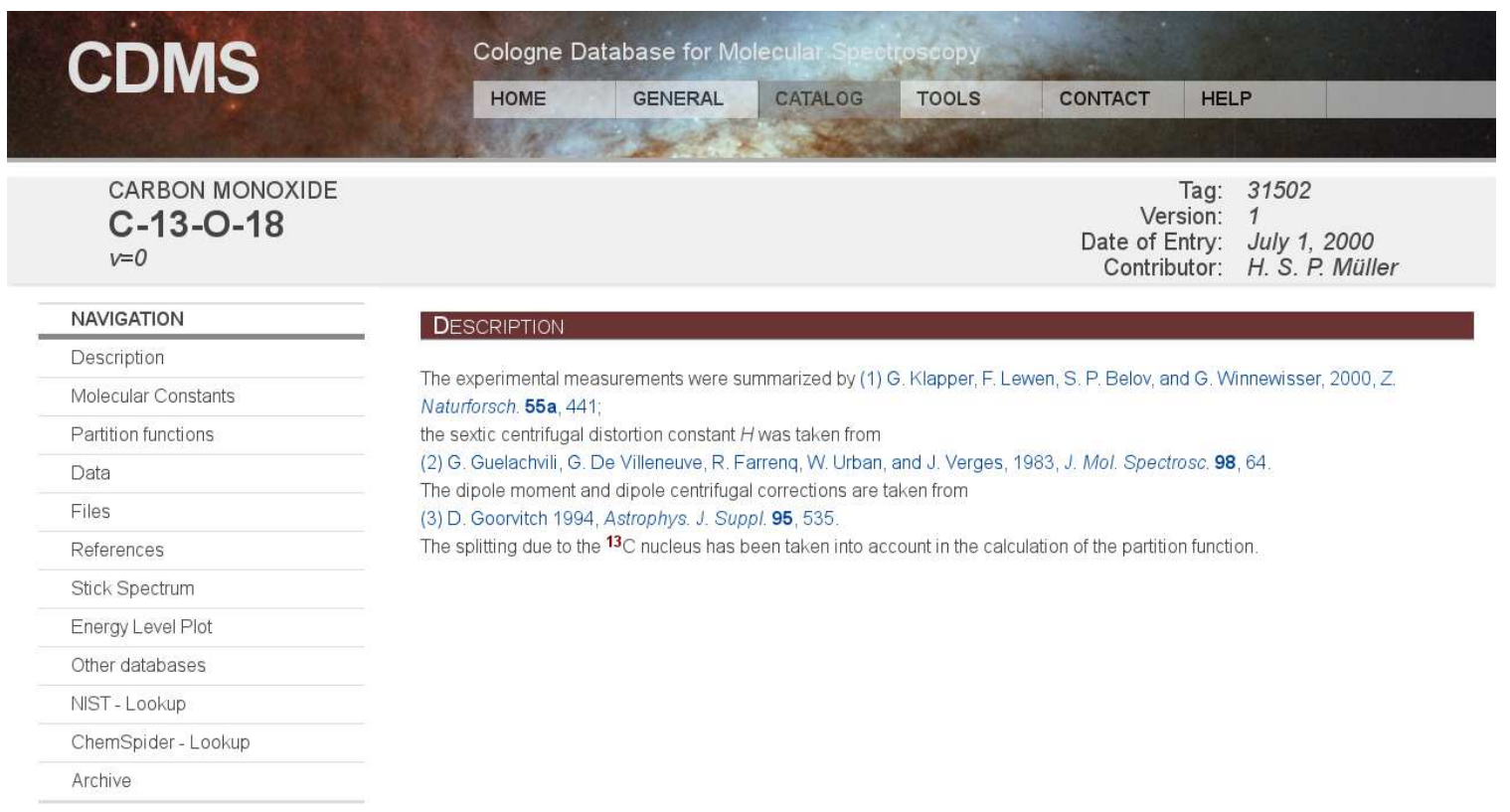

(ㄷ). Physikalisches Institut der Universitât zu Köln

Figure 2: CDMS-Portal - Documentation page of the ${ }^{13} \mathrm{C}^{18} \mathrm{O}$ entry. The page provides basic information on the entry like the date of the entry, version, contributor and a brief description. Further information, such as molecular constants, references, files, and links to other databases can be accessed through the navigation bar on the left.

\subsection{Web-services and libraries}

Currently, there are several web-services, programs and libraries which give users access to the CDMS database. A common web-portal for data retrival has been developed by VAMDC11. It allows users to perform queries to all data and databases in the network and includes basic displaying capabilities. In addition, a new portal for CDMS has been created

http://cdms.ph1.uni-koeln.de/cdms/portal ,

focused on the needs of the user community of the CDMS and JPL databases. Additional information, which cannot be exchanged within the XSAMS document, is implemented here, such as a detailed documentation (see Fig 2), files used in the fit and specific output formats (catalog file format of SPCAT).

Compared to the old CDMS portal, the new version provides extended filter capabilities. Querying all data data for a specific entry (e.g. CO, $v=0$ ), for a specific isotopologue (e.g. ${ }^{13} \mathrm{CO}$ ) or for all isotopologues of a specific molecule (e.g. CO) is possible. Detailed information on states, transitions (see Fig 1) and partition functions are displayed and further restrictions can be posed to limit the number of displayed states to states within a specific energy range or transitions to a specific frequency range. Transitions and states with and without resolved hyperfine structure as well as with and without information on the nuclear spin isomer is shown and filters can be applied accordingly.

A number of software tools are provided by VAMDC 12 which can be applied to query and process data. SpectCol, for example, allows one to combine spectroscopic data with collisional data (e.g. from Basecol). Data can be converted into other output formats such as radex and csv. Several conversion (XSAMS Converter) and visualisation (SpecView) tools can be downloaded after registration. Development of external software packages is also supported. A pythonlibrary (vamdclib) has been developed for this purpose and has been successfully implemented into the XCLASS software package for CASA. It allows one to query and process data via the VAMDC database network and to store

\footnotetext{
${ }^{11}$ www.vamdc.eu/portal

${ }^{12} \mathrm{http}: / /$ www.vamdc.org/activities/research/software/
} 
the data into a local sqlite database. XCLASS [91] uses the CDMS/JPL database to fit astrophysical line spectra in the LTE approximation. It is available for the CASA platform 13 , and its database provides the partition function in 100 steps between 1 and $1000 \mathrm{~K}$, in order to correctly determine the column densities of very low excitation absorption lines.

\section{Summary and outlook}

Over the last years, the CDMS content has been enlarged substantially, and the database has been subject to fundamental infrastructural improvements which makes it now part of the VAMDC database framework. Both measures were necessary steps to make CDMS fit for the use in the ALMA era. The focus of creating further new or updating existing entries will be concerned with species of interest for observations with ALMA or other interferometers. These include larger organic molecules and isotopic or vibrational satellite data of smaller organic molecules along with various radicals, ions, or stable molecules which may be detected in the circumstellar envelopes of young or late-type stars or in external galaxies. We welcome external contributions, in particular for complex and extensive data sets or for entries generated with programs differenr from SPFIT/SPCAT.

The migration of CDMS into VAMDC has been almost completed. The old version of CDMS will be available for some time to serve users who depend on the old ascii format. Based on the large amount of data coming from today's observations automated queries of CDMS alone or in combination with other databases using the http protocol are now possible and encouraged. All users will benefit from the various additional features the database entries as well as the new web portal have. CDMS will further been improved with the aim to keep its role as a very important resource for the interpretation of observations in the millimeter and sub-mm regimes.

\section{Acknowledgements}

We thank the German Ministry of Science (BMBF) for support through contract 05A11PK3. We acknowledge in addition funding by the Deutsche Forschungsgemeinschaft (DFG) through the collaborative research grant SFB 956 "Conditions and Impact of Star-formation", project areas A6, B3, B4, and C3 and through grant Schl 341-15/1.

\section{References}

[1] H.S.P. Müller, S. Thorwirth, D.A. Roth, G. Winnewisser, Astron. Astrophys. 370 (2001) L49-L52.

[2] H.S.P. Müller, F. Schlöder, J. Stutzki, G. Winnewisser, J. Mol. Struct. 742 (2005) 215-227.

[3] H.M. Pickett, R.L. Poynter, E.A. Cohen, M.L. Delitsky, J.C. Pearson, H.S.P. Müller, J. Quant. Spectrosc. Radiat. Transfer 60 (1998) $883-890$.

[4] F.L. Schöier, F.F.S. van der Tak, E.F. van Dishoeck, J.H. Black, Astron. Astrophys. 432 (2005) 369-379.

[5] E. Caux, S. Bottinelli, C. Vastel, J.M. Glorian, The Molecular Universe, Posters from the proceedings of the 280th Symposium of the International Astronomical Union held in Toledo, Spain, May 30-June 3, 2011, \#120.

[6] A.J. Markwick-Kemper, A.J. Remijan, E. Fomalont, Bull. Am. Astron. Soc. 38 (2006) 130.

[7] A.J. Remijan Bull. Am. Astron. Soc. 42 (2010) 568.

[8] N. Jacquinet-Husson, L. Crepeau, R. Armante, C. Boutammine, A. Chédin, et al., J. Quant. Spectrosc. Radiat. Transfer 112 (2011) 2395-2445.

[9] N. Jacquinet-Husson, et al., submitted to J. Mol. Spectrosc., JMS-16-29.

[10] L.S. Rothman, I.E. Gordon, Y. Babikov, A. Barbe, D.C. Benner, et al., J. Quant. Spectrosc. Radiat. Transfer 130 (2013) 4-50.

[11] H.M. Pickett, J. Mol. Spectrosc. 148 (1991) 371-377.

[12] G.L. Pilbratt, J.R. Riedinger, T. Passvogel, G. Crone, D. Doyle, U. Gageur, A.M. Heras, C. Jewell, L. Metcalfe, S. Ott, M. Schmidt, Astron. Astrophys. 518 (2010) Art. No. L1.

[13] Th. de Graauw, F. P. Helmich, T. G. Phillips, J. Stutzki, E. Caux, et al., Astron. Astrophys. 518 (2010) Art. No. L6.

[14] E.T. Young, E.E. Becklin, P.M. Marcum, T.L. Roellig, J.M. De Buizer, et al., Astrophys. J. 749 (2012) Art. No. L17.

[15] S. Heyminck, U.U. Graf, R. Güsten, J. Stutzki, H.W. Hübers, P. Hartogh, Astron. Astrophys. 542 (2012) Art. No. L1.

[16] F. van der Tak, Adv. Space Res. 49 (2012) 1395-1407.

[17] E.F. van Dishoeck, E. Herbst, D.A. Neufeld, Chem. Rev. 113 (2013) 9043-9085.

[18] P. Schilke, D.A. Neufeld, H.S.P. Müller, C. Comito, E.A. Bergin, D.C. Lis, M. Gerin, J.H. Black, M. Wolfire, N. Indriolo, J.C. Pearson, K.M. Menten, B. Winkel, Á. Sánchez-Monge, T. Möller, B. Godard, E. Falgarone, Astron. Astrophys. 566 (2014) Art. No. A29.

[19] M.J. Barlow, B.M. Swinyard, P.J. Owen, J. Cernicharo, H.L. Gomez, R.J. Ivison, O. Krause, T.L. Lim, M. Matsuura, S. Miller, G. Olofsson, E.T. Polehampton, Science 342 (2013) 1343-1345.

\footnotetext{
${ }^{13} \mathrm{http}: / /$ www.astro.uni-koeln.de/projects/schilke/myXCLASSInterface
} 
[20] H.S.P. Müller, S. Muller, P. Schilke, E.A. Bergin, J.H. Black, M. Gerin, D.C. Lis, D.A. Neufeld, S. Suri, Astron. Astrophys. 582 (2015), Art. No. L4.

[21] D.A. Neufeld, E. Falgarone, M. Gerin, B. Godard, E. Herbst, G. Pineau des Forêts, A.I. Vasyunin, R. Güsten, H. Wiesemeyer, O. Ricken, Astron. Astrophys. 542 (2012) Art. No. L6.

[22] S. Brünken, O. Sipilä, E.T. Chambers, J. Harju, P. Caselli, O. Asvany, C.E. Honingh, T. Kamiński, K.M. Menten, J. Stutzki, S. Schlemmer, Nature 516 (2014) 219-221.

[23] O. Asvany, O. Ricken, H.S.P. Müller, M.C. Wiedner, T.F. Giesen, S. Schlemmer, Phys. Rev. Lett. 100 (2008) Art. No. 233004.

[24] F. Wyrowski, K.M. Menten, R. Güsten, A. Belloche, Astron. Astrophys. 518 (2010) Art. No. A26.

[25] K.M. Menten, F. Wyrowski, A. Belloche, R. Güsten, L. Dedes, H.S.P. Müller, Astron. Astrophys. 525 (2011) Art. No. A77.

[26] R. Güsten, L.Å. Nyman, P. Schilke, K. Menten, C. Cesarsky, R. Booth, Astron. Astrophys. 454 (2006) L13-L16.

[27] A. Belloche, R.T. Garrod, H.S.P. Müller, K.M. Menten, Science 345 (2014) 1584-1587.

[28] H.S.P. Müller, A. Coutens, A. Walters, J.-U. Grabow, S. Schlemmer, J. Mol. Spectrosc. 267 (2011), $100-107$.

[29] J. Cernicharo, F. Daniel, A. Castro-Carrizo, M. Agundez, N. Marcelino, C. Joblin, J. R. Goicoechea, M. Guélin, Astrophys. J. 778 (2013) Art. No. L25.

[30] T. Kamiński, C.A. Gottlieb, K.M. Menten, N.A. Patel, K.H. Young, S. Brünken, H.S.P. Müller, M.C. McCarthy, J.M. Winters, L. Decin, Astron. Astrophys. 551 (2013) Art. No. A113.

[31] E. De Beck, W. Vlemmings, S. Muller, J.H. Black, E. OGorman, A.M.S. Richards, A. Baudry, M. Maercker, L. Decin, E.M. Humphreys, Astron. Astrophys. 580 (2015) Art. No. A36.

[32] S. Muller, F. Combes, M. Guélin, M. Gérin, S. Aalto, A. Beelen, J.H. Black, S.J. Curran, J. Darling, D. V-Trung, S. García-Burillo, C. Henkel, C. Horellou, S. Martín, I. Martí-Vidal, K.M. Menten, M.T. Murphy, J. Ott, T. Wiklind, M.A. Zwaan, Astron. Astrophys. 566 (2014) Art. No. A112; and references therein.

[33] E. Roueff, F. Lique, Chem. Rev. 113 (2013) 8906-8938.

[34] F. Dumouchel, J. Kłos, F. Lique, Phys. Chem. Chem. Phys. 13 (2011) 8204-8212.

[35] A. Faure, A.J. Remijan, K. Szalewicz, L. Wiesenfeld, Astrophys. J. 783 (2014) Art. No. 72.

[36] N. Moreau, M.L. Dubernet, ASP Conf. Ser., C. Gabriel, C. Arviset, D. Ponz and E. Solano, eds., 351 (2006) $391-393$.

[37] M.-L. Dubernet, M.H. Alexander, Y.A. Ba, N. Balakrishnan, C. Balança, et al., Astron. Astrophys. 553 (2013) Art. No. 50.

[38] M.L. Dubernet, V. Boudon, J.L. Culhane, M.S. Dimitrijevic, A.Z. Fazliev, et al., J. Quant. Spectrosc. Radiat. Transfer 111 (2010) $2151-2159$.

[39] J. Tennyson, S.N. Yurchenko, Mon. Not. R. Astron. Soc. 425 (2012) 21-33.

[40] V. Wakelam, E. Herbst, J.-C. Loison, I.W.M. Smith, V. Chandrasekaran, et al., Astrophys. J. Suppl. Ser. 199 (2012) Art. No. 21.

[41] D. McElroy, C. Walsh, A.J. Markwick, M.A. Cordiner, K. Smith, T.J. Millar, Astron. Astrophys. 550 (2013) Art. No. 36.

[42] H.S.P. Müller, H. Klein, S.P. Belov, G. Winnewisser, I. Morino, K.M.T. Yamada, S. Saito, J. Mol. Spectrosc. 195 (1999) $177-184$.

[43] H.S.P. Müller, K. Kobayashi, K. Takahashi, K. Tomaru, F. Matsushima, J. Mol. Spectrosc. 310 (2015) 92-98.

[44] T. Amano, Astrophys. J. 716 (2010) L1-L3.

[45] H.S.P. Müller, Astron. Astrophys. 514 (2010) Art. No. L6.

[46] W-F. Thi, F. Ménard, G. Meeus, C. Martin-Zaidi, P. Woitke, E. Tatulli, M. Benisty, I. Kamp, I. Pascucci, C. Pinte, C.A. Grady, S. Brittain, G.J. White, C.D. Howard, G. Sandell, C. Eiroa, Astron. Astrophys. 530 (2011) Art. No. L2.

[47] W.C. Bowman, G.M. Plummer, E. Herbst, F.C. De Lucia, J. Chem. Phys. 79 (1983) 2093-2095.

[48] J.R. Hamilton, A. Faure, J. Tennyson, Mon. Not. R. Astron. Soc. 455 (2016) 3281-3287.

[49] P. Jusko, C. Konietzko, S. Schlemmer, O. Asvany, J. Mol. Spectrosc. 319 (2016) 55-58.

[50] J. Cernicharo, M. Guélin, M. Agúndez, M.C. McCarthy, P. Thaddeus, Astrophys. J. 688 (2008) L84-L86.

[51] J. Pety, P. Gratier, V. Guzmán, E. Roueff, M. Gerin, J.R. Goicoechea, S. Bardeau, A. Sievers, F. Le Petit, J. Le Bourlot, A. Belloche, D. Talbi, Astron. Astrophys. 548 (2012) Art. No. A68.

[52] S. Brünken, L. Kluge, A. Stoffels, O. Asvany, S. Schlemmer, Astrophys. J. 783 (2014) Art. No. L4.

[53] M.C. McCarthy, K.N. Crabtree, M.-A. Martin-Drumel, O. Martinez, Jr., B.A. McGuire, C.A. Gottlieb, Astrophys. J. Suppl. Ser. 217 (2015) Art. No. 10.

[54] H.S.P. Müller, J. Cernicharo, M. Agúndez, L. Decin, P. Encrenaz, J.C. Pearson, D. Teyssier, L.B.F.M. Waters, J. Mol. Spectrosc. 271 (2012), $50-55$.

[55] H.S.P. Müller, J.R. Goicoechea, J. Cernicharo, M. Agúndez, J. Pety, S. Cuadrado, M. Gerin, G. Dumas, E. Chapillon, Astron. Astrophys. 569 (2014), Art. No. L5.

[56] D.T. Halfen, L.M. Ziurys, Astrophys. J. 814 (2015) Art. No. 119

[57] B.J. Drouin, H.S.P. Müller, J. Mol. Spectrosc. 251 (2008) 1-3.

[58] J.C. Pearson, H.S.P. Müller, H.M. Pickett, E.A. Cohen, B.J. Drouin, J. Quant. Spectrosc. Radiat. Transfer 111 (2010) $1614-1616$.

[59] H.M. Pickett, J. Mol. Spectrosc. 228 (2005) 659-663.

[60] H.M. Pickett, J.C. Pearson, C.E. Miller, J. Mol. Spectrosc. 233 (2005) 174-179.

[61] D. Christen, H.S.P. Müller, Phys. Chem. Chem. Phys. 5 (2003) 3600-3605.

[62] H.S.P. Müller, E.A. Cohen, D. Christen, J. Mol. Spectrosc. 216 (2002), 335-344.

[63] R.A. Motiyenko, L. Margulès, E.A. Alekseev, J.-C. Guillemin, J. Demaison, J. Mol. Spectrosc. 264 (2010), 94-99.

[64] H.S.P. Müller, F. Dong, D.J. Nesbitt, T. Furuya, S. Saito, Phys. Chem. Chem. Phys. 12 (2010) 8362-7372.

[65] H.S.P. Müller, A. Belloche, L.-H. Xu, R.M. Lees, R.T. Garrod, A. Walters, J. van Wijngaarden, F. Lewen, S. Schlemmer, K.M. Menten Astron. Astrophys. 587 (2016) Art. No. A92.

[66] L. Kolesniková, B. Tercero, J. Cernicharo, J.L. Alonso, A.M. Daly, B.P. Gordon, S.T. Shipman, Astrophys. J. 784 (2014) Art. No. L7.

[67] S. Yu, B.J. Drouin, J.C. Pearson, H.M. Pickett, Astrophys. J. Suppl. Ser. 180 (2009) 119-124.

[68] H.S.P. Müller, J. Quant. Spectrosc. Radiat. Transfer 130 (2013) 335-340.

[69] C. Medcraft, C.D. Thompson, E.G. Robertson, D.R.T. Appadoo, D. McNaughton, Astrophys. J. 753 (2012) Art. No. 18.

[70] L. Nguyen, A. Walters, L. Margulès, R.A. Motiyenko, J.-C. Guillemin, C. Kahane, C. Ceccarelli, Astron. Astrophys. 553 (2013), Art. No. 
A84.

[71] H.S.P. Müller, D.T. Halfen, L.M. Ziurys, J. Mol. Spectrosc. 272 (2012), 23-26.

[72] I. Kleiner, J. Mol. Spectrosc. 260 (2010), 1-18.

[73] L.-H. Xu, F.J. Lovas, J. Phys. Chem. Ref. Data 26 (1997) 17-156.

[74] C.P. Endres, B.J. Drouin, J.C. Pearson, H.S.P. Müller, F. Lewen, S. Schlemmer, T.F. Giesen, Astron. Astrophys. 504 (2009) 635-640.

[75] L.-H. Xu, R.M. Lees, G.T. Crabbe, J.A. Myshrall, H.S.P. Müller, C.P. Endres, O. Baum, F. Lewen, S. Schlemmer, K.M. Menten, B.E. Billinghurst, J. Chem. Phys. 137 (2012) Art. No. 104313.

[76] J. Fisher, G. Paciga, L.-H. Xu, S.B. Zhao, G. Moruzzi, R.M. Lees, J. Mol. Spectrosc. 245 (2007), 7-20.

[77] M. Carvajal, I. Kleiner, J. Demaison, Astrophys. J. Suppl. Ser. 190 (2010) 315-321.

[78] H. Wang, T.C. Steimle, C. Apetrei, J.P. Maier, Phys. Chem. Chem. Phys. 11 (2009) 2649-2656.

[79] F.J. Lovas, D.F. Plusquellic, B.H. Pate, J.L. Neill, M.T. Muckle, A.J. Remijan, J. Mol. Spectrosc. 257 (2009), 82-93.

[80] D.F. Plusquellic, F.J. Lovas, B.H. Pate, J.L. Neill, M.T. Muckle, A.J. Remijan, J. Phys. Chem. A, 113 (2009) 12911-12918.

[81] Z. Kisiel, O. Dorosh, A. Maeda, I.R. Medvedev, F.C. De Lucia, E. Herbst, B.J. Drouin, J.C. Pearson, S.T. Shipman, Phys. Chem. Chem. Phys. 12 (2010) 8329-8339.

[82] A. Kraśnicki, Z. Kisiel, J. Mol. Spectrosc. 270 (2011), 314-328.

[83] H.S.P. Müller, E.A. Cohen, D. Christen, J. Chem. Phys. 110 (1999) 11865-11875.

[84] D.E. Woon, E. Herbst, Astrophys. J. Suppl. Ser. 185 (2009) 273-288.

[85] H.S.P. Müller, D.E. Woon, J. Phys. Chem. A, 117 (2013) 13868-13877.

[86] J. Tennyson, J. Mol. Spectrosc. 298 (2014), 1-6.

[87] P.J. Mohr, B.N. Taylor, D.B. Newell, Rev. Mod. Phys. 84 (2012) 1527-1605.

[88] H.S.P. Müller, L.R. Brown, B.J. Drouin, J.C. Pearson, I. Kleiner, R.L. Sams, K. Sung, M.H. Ordu, F. Lewen, J. Mol. Spectrosc. 312 (2015), 22-37.

[89] C. Favre, M. Carvajal, D. Field, J.K. Jørgensen, S.E. Bisschop, N. Brouillet, D. Despois, A. Baudry, I. Kleiner, E.A. Bergin, N.R. Crockett, J.L. Neill, L. Margulès, T.R. Huet, J. Demaison, Astrophys. J. Suppl. Ser. 215 (2014) Art. No. 25.

[90] Z. Kisiel, M.-A. Martin-Drumel, O. Piraly, J. Mol. Spectrosc. 315 (2015), 83-91.

[91] T. Möller, C.P. Endres, P. Schilke, Astron. Astrophys. (2015), submitted, arXiv: 1508.04114. 